\title{
References
}

\section{Books and papers}

Amyot, G. (1981), The Italian Communist Party: the crisis of the Popular Front strategy, London: Croom Helm

'Andrea' (1997), 'Intervista', in Del Bello (1997)

Asor Rosa, A. (1977a), Le due società, Turin: Einaudi

Balestrini, N. (1987), Gli invisibili, Milan: Bompiani

Balestrini, N. (1989), L'editore, Milan: Bompiani

Balestrini, N. and P. Moroni (1988), L'orda d'oro, Milan: SugarCo

Balestrini, N. and P. Moroni (1997), L'orda d'oro (revised edition), Milan: Feltrinelli

Becker, H. (1973), Outsiders (enlarged edition), New York: Free Press

Bhaskar, R. (1979), The possibility of naturalism, Brighton: Harvester

Biacchessi, D. (1998), 'Il caso Sofri: cronaca di un'inchiesta', online at www.sofri. org/biacchessi.html (accessed 30/1/2008)

Bobbio, L. (1988), Lotta Continua (second edition), Milan: Feltrinelli

Bonanno, A. (1979), Del terrorismo, di alcuni imbecilli e di altre cose, Catania: Edizioni di 'Anarchismo'

Caselli, G. C. and D. della Porta (1991), 'The history of the Red Brigades: organisational structures and strategies of action (1970-82)', in Catanzaro (1991a)

Catanzaro, R. (ed.) (1991a), The Red Brigades and left-wing terrorism in Italy, London: Pinter

Catanzaro, R. (1991b), 'Subjective experience and objective reality: an account of violence in the words of its protagonists', in Catanzaro (1991a)

Centro di Documentazione 'Fausto e Jaio' del centro sociale Leoncavallo (1995), 'Ipertesto sulle Brigate Rosse', www.ecn.org/leoncavallo/cdoc/br/index.htm, currently offline (accessed 30/4/2005)

Centro d'Iniziativa Luca Rossi (1998), 'Montaldi e l'esperienza proletaria', in L. Parente (ed.), Danilo Montaldi e la cultura di sinistra del secondo dopoguerra, Naples: Città del sole

Chaosmaleont (2001), 'Settantasette', online at http://web.tiscali.it/settanta7/ (accessed 30/1/2008)

Cohen, J. and A. Arato (1992), Civil society and political theory, London: MIT Press 
Cossiga, F. (1997), 'Intervista', in Del Bello (1997)

Davis, H. and P. Walton (1983), 'Death of a premier: consensus and closure in international news', in Davis and Walton (eds), Language, image, media, Oxford: Blackwell

Deaglio, E. (1982), 'Intervista', online at www.xs4all.nl/ welschen/Archief/deaglio. html (accessed 30/1/2008)

Debray, R. (1967) (tr. B. Ortiz), Revolution in the revolution?, London: Pelican

Del Bello, C. (ed.) (1997), Una sparatoria tranquilla: per una storia orale del '77, Rome: Odradek

Della Porta, D. (1995), Social movements, political violence and the state, Cambridge: CUP

Echaurren, P. and C. Salaris (1999), Controcultura in Italia 1967-1977, Boringhieri: Turin

'Enne' (1977), 'Fare fight in Milan', Zero 4, December

Feltri, V. (1994), 'La religione antifascista', in F. Colombo and V. Feltri (1994), Fascismo/Antifascismo, Milan: Rizzoli

Feltrinelli, C. (2001) (tr. A. McEwen), Senior Service, London: Granta

Gamson, W. and D. Meyer (1996), 'Framing political opportunity', in McAdam, McCarthy and Zald (1996)

Giachetta, D. (1997), 'Il movimento del '77 e la violenza', Per il Sessantotto $11-12$

Ginsborg, P. (1990), A history of contemporary Italy, London: Penguin

Goffman, E. (1974), Frame analysis, New York: Harper

Grandi, A. (2003), La generazione degli anni perduti, Turin: Einaudi

Grispigni, M. (1997), 'Elogio degli invisibili', in P. Virno et al., Millenovecentosettantasette, Rome: Manifestolibri

Hellman, S. (1988), Italian Communism in transition, Oxford: OUP

Istituto Carlo Cattaneo (2005), 'Serie storiche degli iscritti ai principali partiti politici italiani dal 1945 al 2004', online at www.cattaneo.org/italiano/archivi/iscritti/ Iscritti.xls (accessed 30/1/2008)

Jamieson, A. (1989), The heart attacked: terrorism and conflict in the Italian state, London: Marion Boyars

Kriesi, H. (1995), 'The political opportunity structure of new social movements: its impact on their mobilisation', in J.C. Jenkins and B. Klandermans (eds) (1995), The politics of social protest, London: UCL

Lumley, R. (1990), States of emergency: cultures of revolt in Italy from 1968 to 1978, London: Verso

Maccari, G. (2000), testimony to Commissione parlamentare d'inchiesta sul terrorismo in Italia e sulle cause della mancata individuazione dei responsabili delle stragi, 21/1/2000; online at www.parlamento.it/parlam/bicam/terror/stenografici/steno60a. htm (accessed 30/1/2008)

Malaspina, T. (1996), 'LC: sette anni di guai', l'Espresso, 5 September

Manconi, L. (1991), 'The political ideology of the Red Brigades', in Catanzaro (1991a) 
Manfredi, G. (2003), 'Archivio anni '70', online at www.gianfrancomanfredi.com/ anni70.html (accessed 30/1/2008)

Massari, R. (1979), Marxismo e critica del terrorismo, Rome: Newton Compton

McAdam, D. (1996), 'Conceptual origins, current problems, future directions', in McAdam, McCarthy and Zald (1996)

McAdam, D., J. McCarthy and M. Zald (eds.) (1996), Comparative perspectives on social movements: political opportunities, mobilising structures, and cultural framings, Cambridge: CUP

Mieli, P. (1984), “'Le Br non esistono”, giurò la sinistra', l'Espresso Archivio, April Miliucci, V. (1997), 'Intervista', in Del Bello (1997)

Monicelli, M. (1978), L'ultrasinistra in Italia 1968-1978, Rome: Laterza

Mordenti, R. (1997), 'Intervista', in Del Bello (1997)

Moroni, P. (1994), 'Origine dei centri sociali autogestiti a Milano', in Francesco Adinolfi et al., Comunitá virtuali. I centri sociali in Italia, Rome: Manifestolibri

Moroni, P. (1996), 'Un certo uso sociale dello spazio urbano', in Consorzio Aaster et al., Centri sociali: geografie del desiderio, Milan: Shake

Morucci, V. (1994), A guerra finita: sei racconti, Rome: Manifestolibri

Moss, D. (1989), The politics of left-wing violence in Italy, 1969-85, London: Macmillan

Napolitano, G., interviewed by E. Hobsbawm (1977), The Italian road to socialism, London: Journeyman

Negri, A. (1978), Il dominio e il sabotaggio, Milan: Feltrinelli

Novaro, C. (1991), 'Social networks and terrorism: the case of Prima Linea', in Catanzaro (1991a)

Piazza, G. (1987), 'Movimenti e sistema politico: il caso di Autonomia operaia' (thesis), Università degli studi di Catania

Piccioni, F. (1997), 'Intervista', in Del Bello (1997)

Pizzo, A. (1997), 'Il carcere e le mie tre vite' (interview with Pasquale Abatangelo), Il Manifesto, 20 April

Potere Operaio (1973), 'Primavalle: incendio a porte chiuse', formerly online at www.tmcrew.org/memoria/primavalle/ (site closed by Polizia Postale 27/5/2003; details at www.tmcrew.org/sequestro.html)

Progetto Memoria (1994), La mappa perduta, Milan: Sensibili alle foglie

Progetto Memoria (1996), Le parole scritte, Milan: Sensibili alle foglie

Rivera, A. (1997), 'Intervista', in Del Bello (1997)

Ruscoe, J. (1982), The Italian Communist Party, 1976-81, London: Macmillan

Salaris, C. (1997), 'Dada dappertutto', in Derive Approdi 15

Sassoon, D. (ed.) (1978), The Italian Communists speak for themselves, Nottingham: Spokesman

Sassoon, D. (1981), The strategy of the Italian Communist Party, London: Frances Pinter

Sassoon, D. (2003), 'Reflection on a death foretold: the life and times of the Italian Communist Party', in R. Leonardi and M. Fedele (eds) (2003), Italy: politics and policy, vol. 2, Aldershot: Ashgate 
Sayer, A. (1984), Method in social science: a realist approach, London: Routledge

Schutz, A. (1962), Collected Papers I: the problem of social reality, The Hague: Martinus Nijhoff

Schutz, A. (1964), Collected Papers II: studies in social theory, The Hague: Martinus Nijhoff

Sciascia, L. (1987) (Italian publication 1978), 'The Moro affair', in L. Sciascia (tr. S. Rabinovitch), The Moro affair and the mystery of Majorana, Manchester: Carcanet

Shore, C. (1990), Italian Communism: the escape from Leninism, London: Pluto Silj, A. (1977), Mai più senza fucile!, Florence: Vallecchi

Snow, D. and R. Benford (1988), 'Ideology, frame resonance, and participant mobilisation', in B. Klandermans, H. Kriesi and S. Tarrow (eds) (1988), From structure to action, London: JAI

Snow, D. and R. Benford (1992), 'Master frames and cycles of protest', in A. Morris and C.M. Mueller (eds), Frontiers in social movement theory, New Haven: Yale University Press

Tarrow, S. (1983), Struggling to reform: social movements and policy change during cycles of protest, Cornell University, Western Societies Paper no. 15

Tarrow, S. (1989), Democracy and disorder: protest and politics in Italy 1965-1975, Oxford: OUP

Tarrow, S. (1991), 'Violence and institutionalisation after the Italian protest cycle', in Catanzaro (1991a)

Tarrow, S. (1996), 'States and opportunities: the political structuring of social movements', in McAdam, McCarthy and Zald (1996)

Tarrow, S. (1998), Power in movement (second edition), Cambridge: CUP

Tilly, C. (1979), 'Repertoires of contention in America and Britain, 1750-1830', in M. Zald and J. McCarthy (eds) (1979), The dynamics of social movements, Cambridge, MA: Winthrop

Tronti, M. (1964), 'Lenin in Inghilterra', Classe operaia 1

Villoresi, L. (1997), 'Così andò quella mattina del 1977, quando Lama . . ', online at www.xs4all.nl/ welschen/Archief/lama.html (accessed 30/1/2008)

Vinciguerra, V. and M. Cipriani (1999), Oppressione, repressione, rivolte: storia d'Italia dal 25 luglio 1943 ad oggi, online at www.fondazionecipriani.it/Kronologia/ introduzione.htm (accessed 30/1/2008)

Virno. P. (1997; originally published 1994), 'Do you remember counter-revolution?', in Balestrini and Moroni (1997)

Vite, F. (2002), “Ma chi ha detto che non c'è”. 1977: storie invisibili' (thesis), Università degli studi di Siena

Wright, S. (1996), 'Negri's class analysis: Italian autonomist theory in the seventies', Reconstruction 8

Wright, S. (1998), 'Missed opportunities - New Left readings of the Italian Resistance', in A. Davidson and S. Wright (eds) (1998), 'Never give in': the Italian Resistance and politics, New York: Peter Lang

Wright, S. (2002), Storming heaven: class composition and struggle in Italian autonomist Marxism, London: Pluto 
Zald, M. (1996), 'Culture, ideology and strategic framing', in McAdam, McCarthy and Zald (1996)

\section{Newspapers}

Anon. (1969a), 'ROMA IN PIAZZA MANIFESTA', l'Unità, 28 February, p1

Anon. (1969b), 'La politica del bastone', l'Unità, 28 February, p1

Anon. (1972a), 'Grave provocazione alla Sit-Siemens di Milano', l’Unità, 4 March, p2

Anon. (1972b), 'I sindacati condannano l'atto teppistico alla Siemens di Milano', l'Unità, 7 March, p4

Anon. (1972c), 'Nel momento più adatto', l'Unità, 17 March, p1

Anon. (1972d), 'La macchina della provocazione', l'Unità, 19 March, p1

Anon. (1972e), 'Aberranti posizioni di un gruppo estremista', l'Unità, 22 March, p6

Anon. (1973a), untitled picture caption, l'Unità, 24 March, p4

Anon. (1973b), 'Torino: bloccata dai 60 mila lavoratori la Fiat Mirafiori', l'Unità (Milan edition), 30 March, p4

Anon. (1973c), 'Un altro episodio della strategia della tensione/Una grava provocazione a Torino: sequestrato un dirigente della FIAT', l'Unità, 11 December, p1

Anon. (1973d), 'Nemici della classe operaia', l'Unità, 11 December, p7

Anon. (1973e), 'Una grande iniziativa del Comune democratico/Trasporti pubblici gratuiti da lunedì a Bologna', l'Unità, 29 March, p1

Anon. (1974a), 'Indegna gazzarra davanti a un negozio Coop', l'Unità (Milan edition), 13 October, p11

Anon. (1974b), 'Una trama evidente', l'Unità, 20 April, p1

Anon. (1974c), 'Sugli omicidi nella sede MSI puntuale la provocazione', l'Unità, 20 June, p1

Anon. (1974d), 'Il comunicato N. 8', l'Unità, 25 May, p5

Anon. (1975a), 'Assemblee, cortei, scioperi in tutto il Paese', l'Unità, 28 September, $\mathrm{p} 2$

Anon. (1975b), 'Il rischio delle mezze verità', Corriere della Sera, 9 July, p1

Anon. (1975c), 'Sulla base della versione fornita dalla polizia sull'uccisione di Anna Maria Mantini/Insufficienti e improvvisate le misure prese per catturare i "nappisti”, l'Unità, 10 July, p5

Anon. (1975d), 'Teppismo e provocazione', l'Unità, 29 September, p2

Anon. (1975e), 'Gli operai cacciano dall'Innocenti un gruppo di provocatori', l'Unità, 30 October, p16

Anon. (1976a), 'Un gesto criminale rivendicato per telefono dalle Brigate rosse/ Reparto della Fiat-Mirafiori distrutto a Torino da un incendio', l'Unità, 4 April, p1

Anon. (1976b), 'Anche l'incendio alla FIAT Rivalta è parte di un piano provocatorio', l’Unità, 15 April, p1

Anon. (1976c), 'Gravissimo episodio dopo l'attacco di un commando all'ambasciata di Spagna/Roma: la polizia spara ed uccide un passante', l'Unità, 15 March, p1 
Anon. (1976d), 'Chi era Francesco Coco', l'Unità, 9 June, p4

Anon. (1976e), 'Nessun alibi', l'Unità, 15 March, p1

Anon. (1976f), 'MANOVRE PROVOCATORIE PREORDINATE DA GRUPPI DI

TEPPISTI/Degenera in violenze e vandalismi il festival di 'Re Nudo' al Parco Lambro', l'Unità (Milan edition), 29 June, p6

Anon. (1976g), 'Il comunicato della segreteria del PCI', l'Unità, 9 June, p1

Anon. (1976h), 'È evidente la mano di un'unica organizzazione terroristica/Stessa tecnica per tutti gli attentati', l'Unità, 15 April, p5

Anon. (1976i), 'Proposte iniziative unitarie di vigilanza', l'Unità, 15 April, p5

Anon. (1976j), 'Due giorni di collettivi e assemblee permanenti alla Statale occupata/ Molta confusione all'happening dei circoli giovanili milanesi', l'Unità, 29 November, p4

Anon. (1976k), “"RAID GASTRONOMICO” DI GIOVANI ESTREMISTI/Mangiano con l'autoriduzione in sette trattorie e pizzerie', Corriere della Sera (Milan edition), 28 June, p16

Anon. (19761), 'Tensione in centro/“Espropriata” salumeria', l’Unità (Milan edition), 28 November, p11

Anon. (1976m), 'I sedicenti "Circoli giovanili", seminando caos e devastazioni, hanno perseguito fino all'ultimo il loro disegno provocatorio', l'Unità (Milan edition), 8 December, p10

Anon. (1976n), 'Il piano dei disordini studiato e preordinato a tavolino dai gruppi teppistici', l'Unità (Milan edition), 8 December, p10

Anon. (1976o), 'Il centro presidiato da migliaia di agenti e carabinieri/Provocazioni e gravi incidenti sconvolgono il centro di Milano per la "prima" alla Scala', l'Unità, 8 December, p1

Anon. (1977a), 'Cinismo e falsa pietà', l’Unità, 5 July, p1

Anon. (1977b), 'Milano: oggi al Lirico le conclusioni di Berlinguer', l'Unità, 30 January, p1

Anon. (1977c), 'Comunicato della Segreteria del PCI', l'Unità, 12 March, p1

Anon. (1977d), 'Gli “autonomi”: una lunga storia di violenze squadristiche', l'Unità, 19 February, p5

Anon. (1977e), 'Roma sconvolta da gravi scontri/Uccisa una ragazza di 19 anni', l'Unità, 13 May, p1

Anon. (1977f), 'L'attacco alla manifestazione', l'Unità, 19 February, p5

Anon. (1977g), 'A cosa mirano le violenze degli “autonomi”/Le provocazioni a Torino contro studenti e operai', l'Unità, 5 March, p1

Anon. (1977h), 'Premeditazione', l'Unità, 22 April, p1

Anon. (1977i), 'Studenti aggrediti e feriti a Torino da squadristi "autonomi” armati', l’Unità, 3 March, p1

Anon. (1977j), 'Condannate a Torino le violenze provocate da bande teppistiche', l'Unità, 5 March, p6

Anon. (1977k), 'A Bologna 150 mila in piazza/Contro-corteo degli studenti', Corriere della Sera, 17 March, p1

Anon. (19771) 'La discriminante democratica è decisiva', l'Unità, 18 March, p1

Anon. (1977m), 'La violenza non vincerà', l'Unità, 7 April, p1 
Anon. (1977n), 'Chiarezza sulla posta in gioco', l'Unità, 28 July, p1

Anon. (1977o), 'Al servizio della reazione', l'Unità, 20 June, p1

Anon. (1977p), 'Il calcolo del terrorismo', l'Unità, 3 June, p1

Anon. (1977q), 'Come rispondere', l'Unità, 17 November, p1

Anon. (1977r), 'Lo stesso disegno', l'Unità, 30 November, p1

Anon. (1977s), 'Tensione e tafferugli tra gli autonomi isolati', l'Unità, 3 December, p6

Anon. (1977t) 'Il documento della Direzione', l'Unità, 20 February, p1

Anon. (1977u), 'I presunti “brigatisti rossi” sorpresi presso Verbania dai carabinieri', l'Unità, 30 April, p5

Asor Rosa, A. (1977b), 'Forme nuove di anticomunismo', l'Unità, 20 February, p3

Barbarisi, G. (1976), 'Tribuna Aperta/Che cosa rispondere all'autoriduzione', Corriere della Sera (Milan edition), 28 November, p15

Berlinguer, E. (1976), 'Il rapporto di Enrico Berlinguer al Comitato centrale e alla Commissione centrale di controllo', l'Unità, 19th October, p8

Berlinguer, E. (1977a), 'Chi sono i nuovi fascisti', La Stampa, 23 September, p1

Berlinguer, E. (1977b), 'Si agita l'anticomunismo per impedire il cambiamento', l'Unità, 19 September, p3

Berlinguer, E. (1977c), 'Con chi non è possibile dialogare', l'Unità, 23 September, p1

Biagi, E. (1977), 'Che guerra è la loro?', Corriere della Sera, 17 November, p1

Borgese, G. (1976), 'MILANO: DECLINO DI UN MITO GIOVANILE/Così muore il festival pop', Corriere della Sera, 29 June, p9

Botta, G. (1977) 'FERMA CONDANNA IN TUTTO IL PAESE/Dell'aggressione squadristica di Roma', l'Unità, 19 February, p1

Bufalini, P. (1977), 'Difesa dell'ordine democratico contro la violenza eversiva', l'Unità, 15 March, p1

Buozzi, G. (1977), 'Radio Alice: sequestrate tutte le apparecchiature', l'Unità, 14 March, p4

Cavallini, M. (1977a), 'Un convegno per tre giorni sul filo di precari equilibri', l'Unità, 26 September, p1

Cavallini, M. (1977b), 'IL CONVEGNO DI BOLOGNA GIRA A VUOTO', l’Unità, 25 September, $\mathrm{p} 1$

Cavallini, M. (1977c), 'Attacchi eversivi alle fabbriche/Come reagiscono gli operai', l’Unità, 21 June, p1

Criscuoli, S. (1977), 'Occorre un fermo impegno unitario contro ogni attacco antidemocratico/ROMA E BOLOGNA SCONVOLTE PER ORE DA ASSALTI DI GRUPPI TEPPISTICI ARMATI', l'Unità, 13 March, p1

d'Adda, G. (1974), 'La Procura: "Si indaga in ogni direzione", Corriere della Sera, 8 October, p2

d'Adda, G. (1975), 'Innocenti: i sindacati prospettano l'occupazione dello stabilimento', Corriere della Sera (Milan edition), 30 October, p12

Enriotti, B. (1977a), 'In un clima sereno ma attento', l'Unità, 23 September, p1

Enriotti, B. (1977b), 'Indetta a Bologna per mercoledì una grande manifestazione unitaria', l'Unità, 14 March, p1 
Enriotti, B. (1977c), 'Finito senza incidenti il raduno di Bologna/È prevalsa la forza della democrazia', l'Unità, 26 September, p1

Fasola, A. (1975), 'Non “prendere” ma conquistare', l'Unità, 29 October, p2

'R. Ga' (1972), 'Gli squadristi alla conquista d'un posto nei circoli "rossi”", l'Unità, 5 March, p7

Ghiara, M. (1977), 'Non basta far tacere le P.38', l'Unità, 20 May, p1

Irdi, L. (1976), 'Assalto ultrà alla' ambasciata di Spagna/La polizia spara, un passante rimane ucciso', Corriere della Sera, 15 March, p1

Lama, L. (1973), 'Una dichiarazione di Lama', l'Unità, 11 December, p7

Lama, L. (1977), 'Il discorso di Luciano Lama', l'Unità, 19 February, p4

'm. m.' (1977), 'SI APRE QUESTA MATTINA A ROMA/Oggi l'assemblea nazionale delle Facoltà in lotta', L'Unità, 26 February, p1

Madeo, A. (1969), 'DIMOSTRAZIONI DI STUDENTI E ATTIVISTI COMUNISTI', Corriere della Sera, 28 February, p1

Maldonado, T. (1977), 'In quale altra città del mondo?', l'Unità, 27 September, p2

Marrocco, M. (1977), 'Due uomini di coraggio', Corriere della Sera, 3 July, p1

Marzullo, K. (1969), 'ROMA IN STATO D'ASSEDIO', l'Unità, 28 February, p2

Marzullo, K. (1977), 'Nel capoluogo emiliano vigorosa risposta popolare alle provocazioni', l'Unità, 13 March, p1

Moncalvo, G. (1977), “Anche il fascismo cominciò con la marcia su Bologna”,, Corriere della Sera, 20 September, p1

Monti, V. (1974), 'Erano ultrà i due banditi ammazzati durante l'assalto in banca a Firenze', Corriere della Sera, 31 October, p11

Monti, V. (1977), 'Ancora incidenti nel capoluogo emiliano', Corriere della Sera, 14 March, p1

Napolitano, G. (1972), 'Responsabilità DC', l'Unità, 21 May, p1

Nava, M. (1976) 'CHI SONO E COSA VOGLIONO I PROTAGONISTI DEL RADUNO/Parlano come pellirossa i giovani arrabbiati alla Statale', Corriere della Sera (Milan edition), 28 November, p16

Padellaro, A. (1977), 'Cossiga preannuncia una legge per chiudere i covi del terrorismo', Corriere della Sera, 8 February, p1 [bylined 'A. Pa.']

Palumbo, A. (1977), 'Tritolo a Milano sui binari del metrò/Fallita l'azione degli autonomi a Roma', l'Unità, 20 May, p1

Paolucci, I. (1977a), 'Una spirale di sanguinosi provocazioni', l’Unità, 20 September, p1

Paolucci, I. (1977b), 'Terrorismo e paura', l'Unità, 5 May, p1

Passanisi, E. (1976), 'Chi vuole turbare la stagione dei contratti', Corriere della Sera, 4 April, p1

Pecchioli, U. (1977), 'Unità contro gli attacchi eversivi', l'Unità, 14 March, p1

Petruccioli, C. (1977a), 'Ricomporre il mosaico', l'Unità, 15 May, p1

Petruccioli, C. (1977b), 'Far vivere una democrazia di massa e andare alle radici della protesta giovanile/GLI INSEGNAMENTI DI BOLOGNA', l'Unità, 27 September, p1

Potere Operaio (1972a), 'Un rivoluzionario è caduto', in Potere Operaio del lunedì, 26 Marcha 
Potere Operaio (1972b), 'Morte di un poliziotto', in Potere Operaio del lunedì, 28 May

Rivolta, C. (1977), “II Lama nel Tibet”, il leader in fuga', in La Repubblica, 19 February

Savioli, A. (1977a), 'Centinaia di migliaia di metalmeccanici e di cittadini alla manifestazione di Roma/UNA FORZA OPERAIA IMMENSA/reclama una svolta politica, economica, morale/e si erge a sicuro baluardo della democrazia', l'Unità, 3 December, $\mathrm{p} 1$

Savioli, A. (1977b), 'Conoscere il terrorismo per poterlo sconfiggere' (interview with Ugo Pecchioli), l'Unità, 14 December, p1

Scagliarini, A. (1977a), 'La città sconvolta per ore dalle violenze', l'Unità, 12 March, p1 [bylined 'a. s.']

Scagliarini, A. (1977b), 'Le indagini confermano: era un piano preordinato', l'Unità, 18 March, p5 [bylined 'a. s.']

Scagliarini, A. (1977c), 'Necessaria l'unità delle forze democratiche contro la spirale delle violenze e delle provocazioni/Gravissimi scontri a Bologna', l'Unità, 12 March, p1

Scagliarini, A. (1977d), 'Bologna: violenze già preordinate dai centri propagandistici eversivi', l'Unità, 19 March, p1 [bylined 'a. s.']

Scagliarini, A. (1977e), 'Ancora scontri ieri sera: 50 arresti', l'Unità, 14 March, p4

Sposito, L. (1973), 'Fanatici della guerriglia urbana', Corriere della Sera, 11 December, p10

Tornabuoni, L. (1977), 'Si sposta sul piano politico la polemica per la guerriglia contro Lama all'università/Il segretario della CGIL: "È nuovo fascismo"”, Corriere della Sera, 19 February, p1

Tortorella, A. (1972), 'La destra e i sui alibi', l’Unità, 19 May, p1

Tortorella, A. (1977a), 'Saper vedere il pericolo', l’Unità, 19 February, p1

Tortorella, A. (1977b), 'Lavoro intellettuale e metodo della libertà', l'Unità, 31 July, p1

Veca, S. (1977), 'Qualcosa di nuovo è in marcia', l'Unità, 27 September, p2 
Phil Edwards - 9781847792921

Downloaded from manchesterhive.com at $04 / 26 / 2023$ 01:19:12PM via free access 